\title{
Synthesis, characterization and properties of a novel silicon- substituted soluble polymer with a PPV structure
}

\author{
Francesco Babudri, ${ }^{\text {a,b }}$ Gianluca M. Farinola, ${ }^{\mathrm{b}}$ Omar Hassan Omar, ${ }^{\mathrm{a}}$ Francesco Naso ${ }^{\mathrm{a}, \mathrm{b}_{*}}$ \\ Chiara Botta, ${ }^{c}$ and Alberto Bolognesi ${ }^{\mathrm{C}}$ \\ ${ }^{a}$ C.N.R. Istituto di Chimica dei Composti OrganoMetallici I.C.C.O.M. Sezione di Bari, \\ Dipartimento di Chimica, Università di Bari, via Orabona 4, I-70126 Bari, Italy \\ ${ }^{b}$ Dipartimento di Chimica, Università di Bari, via Orabona 4, I-70126 Bari, Italy \\ ${ }^{c}$ CNR Istituto per lo Studio delle Macromolecole, via Bassini 15, I-20133 Milan, Italy \\ E-mail: naso@area.ba.cnr.it
}

Dedicated to Prof. Domenico Spinelli on his $70^{\text {th }}$ birthday

(received 18 Oct 02; accepted 14 Jan 03; published on the web 22 Jan 03)

\begin{abstract}
Soluble poly(2,5-di-t-butyldimethylsilyloxy-1,4-phenylenevinylene) was prepared via a Stille coupling reaction between 2,5-diiodo-1,4-bis-( $t$-butyldimethylsilyloxy)benzene and E-1,2-bis(tri$n$-butylstannyl)ethylene in the presence of tetrakis-(triphenylphosphine)palladium(0) as the catalyst. The characterization of this material by ${ }^{1} \mathrm{H}$ - and ${ }^{13} \mathrm{C}-\mathrm{NMR}$, UV-Vis., and IR spectroscopies is described. The molecular weights, determined by GPC analysis and referred to standard polystyrene, were $M_{n}=2027$ and $M_{w}=2621$. Photoluminescence measurements were performed in solution and on thin films of pure material or of a blend with polystyrene.
\end{abstract}

Keywords: Conjugated polymer, poly(p-phenylenevinylene), PPV, Stille reaction

\section{Introduction}

In recent years, much interest devoted to conjugated polymers has been elicited by the possibility of employing them as active elements in the fabrication of semiconductor devices. ${ }^{1}$

Poly(phenylenevinylene) (PPV) polymers appear among the most promising materials for manufacturing electroluminescent devices, ${ }^{2}$ owing to their good structural properties combined with the possibility of modulating the band gap and the light emission spectra by introducing various substituents on the aromatic rings. ${ }^{2 c}$ Although the color-tunability over the full visible range could be obtained by control of the HOMO-LUMO band-gap of the materials, the emission in the blue region of the spectrum has proved to be a challenge, and organic blueemitters are considered an important focus of technological interest. ${ }^{3}$ 
In blue-fluorescing conjugated polymers, the semiconductor band-gap is wider than in currently known processable dialkoxy PPVs. ${ }^{3}$ One commonly employed strategy for achieving the enlargement of the band-gap in PPV polymers is represented by the reduction of the so-called effective conjugation length. The presence of groups that interrupt the conjugation along the polymer main chain, or the introduction of sterically demanding substituents that increase the torsion angle around the vinylene linkage, can be employed as means to achieve this result. In both these approaches the use of silyl substituents has been shown to play an important role, either as spacers in the polymer main chains, ${ }^{4}$ or as bulky substituents which slightly break the coplanarity of the conjugated backbone. ${ }^{5}$ The electron-withdrawing effect of the organosilicon substitution has also been invoked to rationalize the observed enlargement of the PPV band gap in a $t$-butyldimethylsilyloxy-substituted alkoxy PPV. ${ }^{6}$

Various synthetic approaches have been described in the literature for the preparation of PPV derivatives bearing silicon substituents, for example, Wittig olefination, ${ }^{4 \mathrm{~b}}$ the Wessling procedure with sulfonium salts, ${ }^{5 a}$ or dehydrohalogenation of p-xylylene dihalides. ${ }^{5 b, 6}$ In previous work we have conveniently prepared a number of low-molecular-weight PPV derivatives by using the Stille cross-coupling reaction, ${ }^{7}$ starting from suitable di-iodoaryl derivatives and $E$-1,2-bis(tri- $n$ butylstannyl)ethylene. ${ }^{8}$ The investigations performed on the resulting materials have shown that, in spite of the low molecular weight, they display photophysical properties of special interestgiving further evidence that oligomers can be used, beside high molecular weight materials, in optical and electro-optical applications. ${ }^{8 c, 9}$ Now, we report an extension of this methodology to the synthesis of a sterically hindered dialkoxysilyl- substituted PPV, together with the characterization and some relevant properties of the obtained material.

\section{Results and Discussion}

\section{Synthesis and characterization}

The cross-coupling reaction of 2,5-di-iodo-1,4 bis(t-butyldimethylsilyloxy)benzene (3) and E1,2-bis(tri- $n$-butylstannyl)ethylene (4), depicted in Scheme 1 , was carried out using tetrakis(triphenylphosphine)palladium(0) as catalyst, in boiling benzene (see Experimental Section); both monomers were used in equimolecular amounts.

Monomer (3) was obtained by silylation of the 2,5-di-iodo-1,4-dihydroxybenzene (2) prepared by a demethylation reaction by boron tribromide ${ }^{10}$ of the dimethyl ether (1), which in turn was obtained following a procedure reported for similar compounds. ${ }^{11}$

Average molecular weights of the polymer (5) were $M_{n}=2621$ and $M_{w}=2027$ with a $M_{w} / M_{n}$ ratio of 1.29, determined by Gel Permeation Chromatography (G.P.C.) analysis on the basis of monodisperse polystyrene standards, using THF as solvent.

The ${ }^{1} \mathrm{H}$-NMR spectrum of polymer 5 shows broad resonances centered at $\delta 7.24 \mathrm{ppm}$ (aromatic protons), $7.06 \mathrm{ppm}$ (vinyl protons), $1.03 \mathrm{ppm}$ (t-butyl groups), and $0.21 \mathrm{ppm}$ (methyl groups on the silicon atoms). In the ${ }^{13} \mathrm{C}$ NMR spectrum the following resonances are detectable: 
$\delta 147.71 \mathrm{ppm}$ (aromatic carbon atoms linked to the oxygen atoms); $128.64 \mathrm{ppm}$ (aromatic carbon atoms linked to the C-C double bonds); $122.52 \mathrm{ppm}$ (vinyl carbon atoms); $115.94 \mathrm{ppm}$ (C-H aromatic carbon atoms); 25.94, 18.26, -4.12 ppm (alkyl carbon atoms).

The band at $941 \mathrm{~cm}^{-1}$, which is assigned to the out-of-plane bending of the vinyl C-H bond in the FT-IR spectrum, is in accordance with a trans- geometry of the double bonds. Other relevant bands were assigned to the alkyl-silicon-oxygen linkage (band at $1132 \mathrm{~cm}^{-1}$ ) or to alkyl C-H stretching (bands at 2956, 2929 and $2858 \mathrm{~cm}^{-1}$ ).

In order to ascertain the nature of the chain terminations we performed an XPS analysis of the polymer $\mathbf{5}$ for evaluating the tin and iodine contents. This analysis revealed low values of atomic concentrations for both of these elements (under $0.5 \%$ ), very close to the detection threshold.

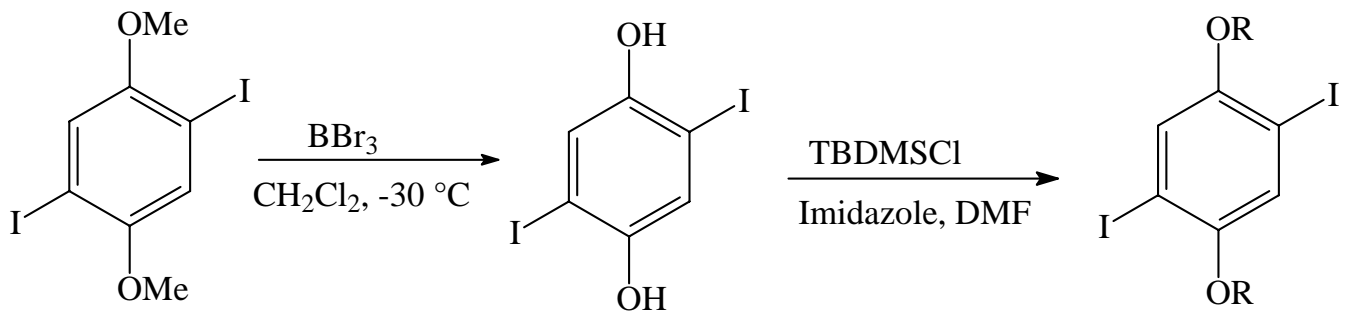

1

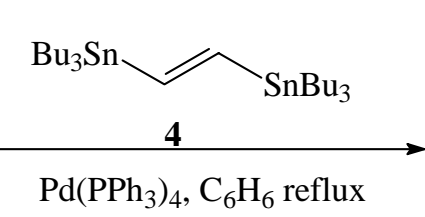

2

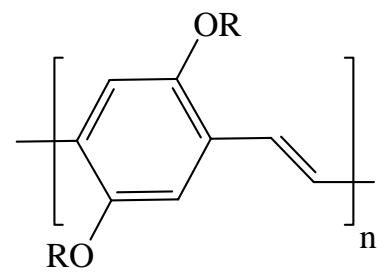

$5 \mathrm{R}=$ TBDMSi

Scheme 1. Synthesis of the aromatic monomer 3 and polymerization reaction.

\section{Optical properties}

The optical properties of polymer $\mathbf{5}$ have been investigated by means of UV-Vis spectroscopy and photoluminescence (PL) measurements.

The absorption spectra, in chloroform solution, of polymer 5 and of poly[2,5-bis(1pentyloxy)-1,4-phenylene vinylene] previously prepared by the same procedure, ${ }^{8 \mathrm{a}}$ are reported in Figure 1. The absorption maximum for polymer $5(451 \mathrm{~nm})$ is slightly blue-shifted with respect to that of the alkoxy- substituted PPV (465 nm). As discussed above, this may be ascribed to the steric effect of the two bulky dimethyl- $t$-butylsilyl groups in comparison with the alkoxy linear chains of the previously described PPV. 


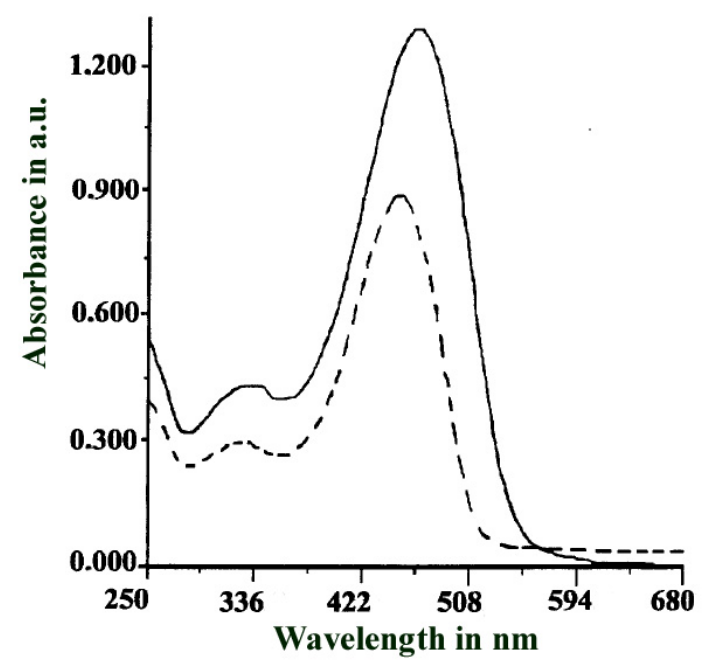

Figure 1. Absorption spectra of poly[2,5-bis(1-pentyloxy)-1,4-phenylene vinylene] (solid line) and of polymer 5 (dotted line).

The PL spectrum in THF solution is reported in Figure 2, together with the PL spectrum in a blend of polymer 5/Polystyrene (PS) (5/PS ratio 80/20).

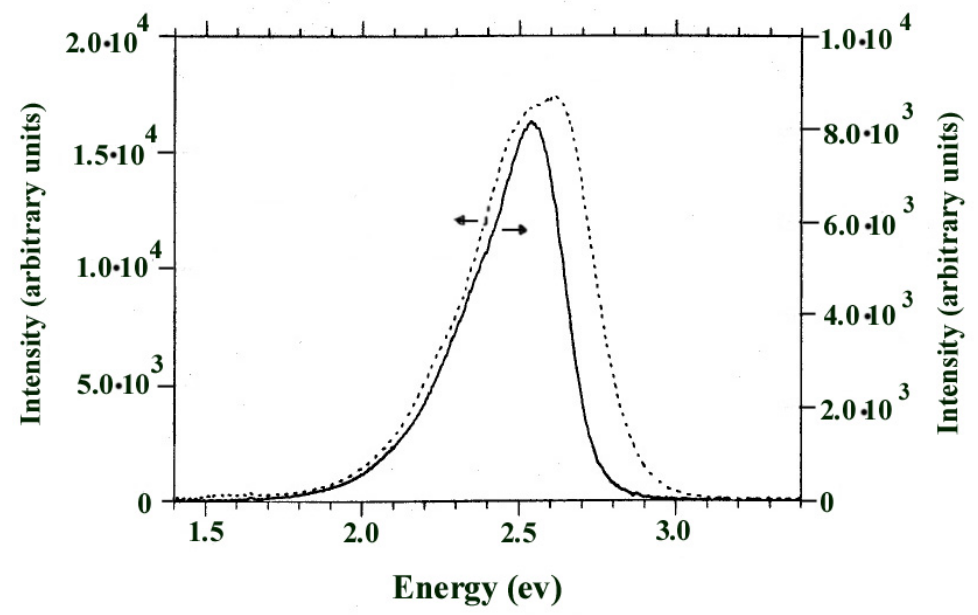

Figure 2. Photoluminescence spectra of polymer $\mathbf{5}$ in chloroform solution (dotted line) and in blend with polystyrene (solid line).

As can be clearly seen, the two spectra are almost equivalent (2.54 eV and $2.59 \mathrm{eV}$ are the maxima related to the blend- and solution spectra, respectively, in the green region). A quantum yield of $18.5 \%$ has been measured in dilute THF solution. On the other hand, the PL of a cast 
film of 5 was not measurable, owing to its very low intensity. Similarly, only the blend was able to give electroluminescence in an LED formed by ITO/polymer 5-PS/Al, with an external quantum efficiency of $0.005 \% .{ }^{12}$ In our opinion, this is a clear indication that the solid-state aggregation strongly improves the exciton migration towards quenching sites, while in the blend, as in solution, the large interchain distance improves exciton intra-chain radiative recombination and consequently the PL values are controlled by the distance among the chromophores.

In principle, the lack of PL in the solid state could be attributed to an ordered phase in 3-D. Surprisingly, even after prolonged annealing, the polymer did not show any endothermic peak in the DSC scan, nor any detectable birefringence in the optical microscope in polarized light. Therefore, it seems reasonable to suggest that, even though the polymer is in its amorphous state, the interchain distances are short enough to promote exciton migration among the chains. The short interchain distances in this polymer seem to be due to the reduced lengths of the -O-Si$\mathrm{C}\left(\mathrm{CH}_{3}\right)_{3}$ group with respect to other $n$-alkoxy-substituted PPV, where higher interchain distances provide a higher PL efficiency in the solid state. ${ }^{4 a, 9 a, 13}$

\section{Conclusions}

A novel silyloxy-substituted PPV has been prepared by the Stille cross-coupling reaction. This enhances the synthetic potential of such a reaction for the ready stereoselective preparation of members of the PPV family.

The introduction of the silyloxy group is responsible for a blue shift of the absorption spectrum of the polymer with respect to alkoxy-substituted PPVs.

The PL efficiency in solution is $18.5 \%$, while in the solid state it is very low - in agreement with other silicon containing PPV. ${ }^{4 a}$ Blends with polystyrene have much higher quantum yields, making this material a possible candidate for the preparation of multicomponent LEDs with emission maxima shifted towards the blue region of the visible spectrum.

\section{Experimental Section}

General procedures. The dimethyl ether, $\mathbf{1}$, and monomer, $\mathbf{4}$, were prepared as reported. ${ }^{10,11}$ Tetrakis-(triphenylphosphine)- palladium(0) was a commercial product. Benzene was distilled immediately prior to use from sodium/benzophenone in a nitrogen atmosphere. ${ }^{1} \mathrm{H}$ - NMR and ${ }^{13} \mathrm{C}$ - NMR spectra were recorded in $\mathrm{CDCl}_{3}$ at $500 \mathrm{MHz}$ and $125 \mathrm{MHz}$, respectively, on a Bruker AM 500 spectrometer, using the residual $\mathrm{CHCl}_{3}$ as the standard for the ${ }^{1} \mathrm{H}$ data and the triplet centered at $\delta 77.00$ for the ${ }^{13} \mathrm{C}$ data. UV-Visible spectra were recorded with a Varian DMS 100 spectrometer in $\mathrm{CHCl}_{3}$ solution. FT-IR spectra were registered in $\mathrm{KBr}$ pellets on a Perkin-Elmer 1710 spectrometer. Molecular weights were determined with a Waters 600E instrument using THF as a solvent. DSC experiments were obtained on a Perkin Elmer DSC-2C instrument under 
a nitrogen atmosphere at $20^{\circ} \mathrm{C} / \mathrm{min}$. PL spectra were recorded with a SPEX $270 \mathrm{M}$ spectrometer equipped with a liquid- $\mathrm{N}_{2}$ - cooled CCD detector. The quantum yield was measured with respect to quinine disulfate standard solutions.

2,5-di-Iodo-1,4-dihydroxybenzene (2). A solution of boron tribromide (25.3 $\mathrm{mL} 1 \mathrm{M}$ solution in $\mathrm{CH}_{2} \mathrm{Cl}_{2}, 25.3 \mathrm{mmol}$ ) was added at $-80{ }^{\circ} \mathrm{C}$ to a stirred solution of 1,4-di-iodo-2,5dimethoxybenzene 1 ( $4.49 \mathrm{~g}, 11.51 \mathrm{mmol})$ in $\mathrm{CH}_{2} \mathrm{Cl}_{2}(45 \mathrm{~mL})$. The reaction mixture was slowly warmed up to room temperature and quenched with water $(100 \mathrm{~mL})$. The organic phase was separated and the aqueous phase extracted with $\mathrm{CH}_{2} \mathrm{Cl}_{2}(2 \times 50 \mathrm{~mL})$. The combined organic phases were dried over anhydrous sodium sulfate and the solvent evaporated at reduced pressure. A white solid was obtained (3.66 g, $88 \%$ yield), m.p. $204-206{ }^{\circ} \mathrm{C}$ (ethanol / water). ${ }^{1} \mathrm{H}$ - NMR $\left(\mathrm{CDCl}_{3}\right) \delta 4.93$ (bs, 2H); 7.27 (s, 2H).

2,5-di-Iodo-1,4-bis(t-butyldimethylsilyloxy)benzene (3). A mixture of 2 (1.40 g, $3.86 \mathrm{mmol}$ ), t-butyldimethylsilyl chloride (1.40 g, $9.29 \mathrm{mmol})$ and imidazole (1.32 g, $19.31 \mathrm{mmol})$ in DMF (2 $\mathrm{mL}$ ) was stirred at room temperature for $1 \mathrm{~h}$, then diluted with water $(50 \mathrm{~mL})$ and extracted with ethyl acetate $(3 \times 50 \mathrm{~mL})$. The organic phase was washed with aqueous hydrochloric acid ( $1 \mathrm{M}$, $50 \mathrm{~mL}$ ), then with water ( $3 \times 50 \mathrm{~mL}$ ) and dried over anhydrous sodium sulfate. After evaporation of the solvent at reduced pressure, the crude product was purified by flash chromatography (elution with petroleum). A white solid was obtained (1.91 g, $84 \%$ yield) m.p. $114-115{ }^{\circ} \mathrm{C}$ (ethanol). IR (KBr) 2957, 2944, 2927, 2855, 1473, 1349, 1257, 1216, $913 \mathrm{~cm}^{-1} .{ }^{1} \mathrm{H}$ NMR $\left(\mathrm{CDCl}_{3}\right) \delta 7.15$ (s, 2H); 1.02 (s, $\left.18 \mathrm{H}\right) ; 0.24$ (s, $\left.12 \mathrm{H}\right) \mathrm{ppm} .{ }^{13} \mathrm{C} \mathrm{NMR}\left(\mathrm{CDCl}_{3}\right) \delta$ 150.20; 127.82; 89.45; 25.85; 18.31; -4.10 ppm.

Poly[2,5-di(t-butyldimethylsilyloxy)-1,4-phenylene vinylene] (5). A mixture of 2,5-di(t-butyldimethylsilyloxy)-1,4-di-iodobenzene 3 ( $2.36 \mathrm{~g}, 4.00 \mathrm{mmol}$ ), E-1,2-bis(n-tributylstannyl)ethylene 4 (2.42 g, $3.98 \mathrm{mmol}$ ) and tetrakis(triphenylphosphine)palladium(0) (0.56 g, 0.48 $\mathrm{mmol}$ ), in $95 \mathrm{ml}$ of anhydrous benzene was heated under reflux under a nitrogen atmosphere. After 3 days a dark green precipitate slowly separated from the reaction mixture. After 10 days the mixture was cooled to room temperature, the solvent was evaporated under reduced pressure, and the residue was diluted with water and extracted with $\mathrm{CH}_{2} \mathrm{Cl}_{2}(3 \times 50 \mathrm{~mL})$. The crude polymer obtained by evaporating the solvent, was washed thrice with hexane; the solid was recovered by centrifugation after each washing. Low molecular weight fractions were eliminated by extraction in a Soxhlet apparatus with ethanol for 24 hours. The yellow-green powder $(0.58 \mathrm{~g}$, $40 \%$ yield) was dried at $90^{\circ} \mathrm{C}$ and $10^{-2}$ mbar. The polymer is soluble in $\mathrm{CH}_{2} \mathrm{Cl}_{2}, \mathrm{CHCl}_{3}$ and THF, insoluble in ethanol.

\section{Acknowledgements}

This work was financially supported by Ministero dell'Università e della Ricerca Scientifica e Tecnologica, Rome (Project "Sintesi di materiali organici per applicazioni ottiche" L. 488 
19/12/92, Piano "Materiali Innovativi" and National Project "Stereoselezione in Sintesi Organica. Metodologie ed Applicazioni"), and by the University of Bari.

\section{References}

1. Hadziioannou, G.; van Hutten, P.F. Eds.; Semiconducting Polymers; Wiley-VCH: Weinheim, 2000.

2. (a) Burroughes, J.H.; Bradley, D.D.C.; Brown, A.R.; Marks, R.N.; Mackay, K.; Friend, R.H.; Burns, P.L.; Holmes, A.B. Nature 1990, 347, 539. (b) Braun, D.; Heeger, A.J. Appl. Phys. Lett. 1991, 58, 1982. (c) Burn, P.L.; Holmes, A.B.; Kraft, A.; Bradley, D.D.C.; Brown, A.R.; Friend, R.H.; Gymer, R.W. Nature 1992, 356, 47. (d) Gustafsson, G.; Cao, Y.; Treacy, G.M.; Klavetter, F.; Colaneri, N.; Heeger, A.J. Nature 1992, 357, 477. (e) Greenham, N.C.; Moratti, S.C.; Bradley, D.D.C.; Friend, R.H.; Holmes, A.B. Nature 1992, 365, 628. (f) Son, S.; Dodabalapur, A.; Lovinger, A.J.; Galvin, M.E. Science 1995, 269, 376. (g) Strukelj, M.; Miller, T.M.; Papadimitrakopoulos, F.; Son, S. J. Am. Chem. Soc. 1995, 117, 11976. (h) Kraft, A.; Grimsdale, A.C.; Holmes, A.B. Angew. Chem. Int. Ed. 1998, 37, 402.

3. Kim, D.Y.; Cho, H.N.; Kim, C.Y. Prog. Polym. Sci. 2000, 25, 1089.

4. Garten, F.; Hilberer, A.; Cacialli, F.; Esselink, E.; van Dam, Y.; Schlatmann, B.; Friend, R.H.; Klapwijk, T.M.; Hadziioannou, G. Adv. Mater. 1997, 9, 127; (b) Kim, H.K.; Ryu, MK.; Lee, S-M. Macromolecules 1997, 30, 1236; (c) Kim, H.K.; Ryu, M.-K.; Kim, K.-D.; Lee, S.-M.; Cho, S.W.; Park, J.-W. Macromolecules 1998, 31, 1114; (d) Kim, K.-D.; Park, J.S.; Kim, H.K.; Lee, T.B.; No, K.T. Macromolecules 1998, 31, 7267; (e) Jung, S-H.; Kim, H.K.; Kim, S.-H.; Kim, Y.H.; Jeoung, S.C.; Kim, D. Macromolecules 2000, 33, 9277.

5. (a) Hwang, D-H.; Shim, H-K.; Lee, J-I.; Lee, K-S. J. Chem. Soc., Chem. Commun. 1994, 2461. (b) Hwang, D-H.; Kim, S.T.; Shim, H-K.; Holmes, A.B.; Moratti, S.C.; Friend, R.H. J. Chem. Soc., Chem. Commun. 1996, 2241.

6. Höger, S.; McNamara, J.J.; Schricker, S.; Wudl, F. Chem. Mater. 1994, 6, 171.

7. (a) Stille, J. K., Angew. Chem. 1986, 98, 504. Angew. Chem., Int. Ed. 1986, 25, 508. (b) Mitchell, T.N. Synthesis 1992, 803.

8. (a) Babudri, F.; Cicco, S.R.; Farinola, G.M.; Naso, F.; Bolognesi, A.; Porzio, W. Macromol. Rapid Commun., 1996, 17, 905. (b) Naso, F.; Babudri, F.; Farinola, G.M. Pure Appl. Chem., 1999, 71, 1485. (c) Babudri, F.; Cicco, S.R.; Chiavarone, L.; Farinola, G.M.; Lopez, L.C.; Naso, F.; Scamarcio, G. J. Mater. Chem. 2000, 10, 1573. (d) Babudri, F.; Cardone, A.; Chiavarone, L.; Ciccarella, G.; Farinola, G.M.; Naso, F.; Scamarcio, G. Chem. Commun. 2001, 1940.

9. (a) Chiavarone, L.; Di Terlizzi, M.; Scamarcio, G.; Farinola, G.M.; Babudri, F.; Naso, F. Appl. Phys. Lett., 1999, 75, 2053. (b) Cassano, T.; Tommasi, R.; Ferrara, M.; Babudri, F.; Farinola, G. M.; Naso, F. Linear, Nonlinear and Power-Limiting Organics, Eich, M.; 
Kuzyk, M.G.; Lawson, C.M.; Norwood, R.A. Eds.; Proc. SPIE 2000; 4106, 395. (c) Cassano, T.; Tommasi, R.; Ferrara, M.; Babudri, F.; Farinola, G.M.; Naso, F. Chem. Phys.

10. 2001, 272, 111. (d) Cassano, T.; Tommasi, R.; Babudri, F.; Cardone, A.; Farinola, G.M.; Naso, F. Optic Letters 2002, in press.

11. Vickery, E.H.; Pahler, L.F.; Eisenbaum, E.J., J. Org. Chem. 1979, 44, 4444.

12. Bao, Z.; Chen, Y.; Cai, R.; Yu, L. Macromolecules 1993, 26, 5281.

13. Bolognesi, A.; Botta, C.; Babudri, F.; Farinola, G. M.; Hassan Omar, O.; Naso, F. Synth. Met. 1999, 102, 919.

14. Doi, S.; Kuwabara, M.; Noguchi, T.; Onoshi, T. Synth. Met. 1993, 57, 4174. 